\title{
Dual Band High Directivity Microstrip Patch Antenna Rotated- Stepped-Impedance Array Loaded with CSRRs for WLAN Applications
}

\author{
Deepak, Dr. Jaswinder Kaur \\ Department of Electronics and Communication Engineering, Thapar University, Patiala, 147004 India
}

\begin{abstract}
A suspended dual band planar antenna based on a stepped-impedance structure modified to an array, loaded with Complementary Split-Ring Resonators (CSRRs), possessing good directivity pertaining point to point backhaul communication is acquainted. The presented antenna radiates at $2.4-2.5 \mathrm{GHz}(4.4 \%)$ and $5.3-5.9 \mathrm{GHz}$ $(12 \%)$ with $\left|S_{11}\right|<-10 \mathrm{~dB}$, accompanying directivity of $11.6 \mathrm{dBi}$ and $11.3 \mathrm{dBi}$ respectively. The antenna is loaded with CSRRs which works as a L-C tank resonator, to obtain better coupling, and to lower the frequency of radiation in $2.4 \mathrm{GHz}$ band along with decreasing the return loss for both the bands of WLAN. The Suspended antenna, symmetrical across vertical axis, has four branches, each of which are obtained from Maximally Stepped-Impedance filter. A stable radiation pattern with high directivity in both the WLAN bands is achieved.
\end{abstract}

Index Terms: Antennas, array, CSRR, dual-band, filter, metamaterial, microstrip antennas, miniaturized antennas, multi-frequency antennas, patch antennas, planar, resonators, SRR, Stepped-Impedance, WiFi, WLAN.

(C) 2016 Published by MECS Publisher. Selection and/or peer review under responsibility of the Research Association of Modern Education and Computer Science

\section{Introduction}

PLAIN profile antennas have been exploited for point to point backhaul communication links for a long time. They exhibit a profile lower than that of horn, cavity backed, and reflector antennas. Reduced Q-value, loss tangent, and lower cost have been observed with suspended patch antennas, where the substrate's material is replaced by air. As a result, lower dielectric, surface wave losses with better bandwidth has been achieved, at the cost of increased height $.01 \lambda-.1 \lambda$, where $\lambda$ is the free space wavelength.

Industrial, Scientific, and Medical (ISM) bands, unlicensed bands, are very popular for the obvious reason,

* Corresponding author.

E-mail address: deepak.08.wc@gmail.com, jaswinder.kaur@thapar.edu 
no need to get license or pay for the same. IEEE $802.11 \mathrm{a} / \mathrm{b} / \mathrm{g} / \mathrm{n}$ [1] WLAN standards, operate in this band, namely $2.4 \mathrm{GHz}$ band with frequency range $2.4-2.485 \mathrm{GHz}$, and $5 \mathrm{GHz}$ bands with frequency range 5.150$5.350 \mathrm{GHz}, 5.470-5.725 \mathrm{GHz}$, and 5.725-5.850 GHz. Bandwidth of channels varying from $5 \mathrm{MHz}$ to $20 \mathrm{MHz}$ within each band. Usually, for point-to-point communications, 2.4 and $5 \mathrm{GHz}$ antennas are mounted separately, excited through discrete ports, whereas, Broadband Planar antennas, usually, does have a stable directional pattern, for this complete range. Therefore, a dual band Patch [2][3][4] can be used for its simple configuration, low profile, and configurable directional patterns. This paper exhibits a Dual Band Microstrip Patch antenna loaded with Complementary Split-Ring Resonators (CSRRs) [5] for WLAN applications.

\section{Design Methodology}

While designing this microstrip patch antenna, following problems have been taken into consideration:

1) Overlapping of radiation of individually placed antennas for $2.4 \mathrm{GHz}$ and $5.5 \mathrm{GHz}$, the presence of coupling between them results in reduction in radiation efficiency.

2) Electrical length of antennas (for $2.4 \mathrm{GHz} \& 5.5 \mathrm{GHz}$ ) are different, this $2.4 \mathrm{GHz}$ radiator has large electrical length at $5.5 \mathrm{GHz}$, higher order modes from $5.5 \mathrm{GHz}$ resonances with $2.4 \mathrm{GHz}$ radiation, giving out unwanted radiation patterns.

3) Separate single port feeding is required for each radiating antenna.

4) Directivity of the antenna should be high.

5) Material costs should be low, so that the antenna can be easily used commercially.

The proposed solutions are as follows:

1) Series fed network is used, reducing the count of microstrip lines, minimizing the unwanted mutual coupling between various feeding networks and radiators. Also Split Ring Resonators (SRRs) are employed to manipulate the coupling further.

2) Microstrip Stepped-Impedance low pass filter is used such that it has a passband over $2.4 \mathrm{GHz}$ and stopband over $5.5 \mathrm{GHz}$, to excite the $2.4 \mathrm{GHz}$ radiator. This avoids $2.4 \mathrm{GHz}$ radiations from showing resonance in $5.5 \mathrm{GHz}$ band. This hi-Z low-Z low pass filter is easy to design and occupy less space than that used with stubs, but this does not produce a sharp attenuation, which is not needed here as both the bands are not consecutive but far apart.

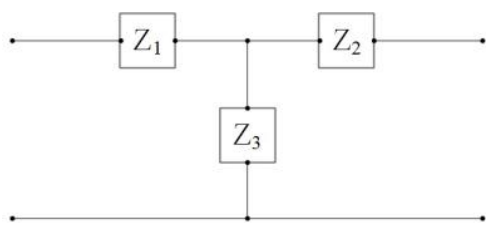

(a)

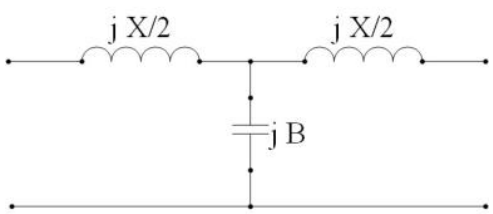

(b)

Fig.1.(a) General Two-Port T-Network. (b) Equivalent circuit for short Transmission-Line 
3) Signals are reflected through resonators, and then radiated again. Better impedance matching is employed, adding more control over parameters through Split Ring Resonators.

4) For better directivity, design is a modification over an array [6], where arrays are always known to enhance the directivity.

5) This design is a suspended patch antenna design, thus air is used as a substitute for substrate material, So that the material cost for substrate is very less, which can play a big role in commercial use.

\section{Design of Feeding Network, Filter, and Split Ring Resonators}

Here, distributed elements have been used for designing the filters, with significant distances between them. Lumped Circuit Models can not predict accurately the filter response of the Distributed circuit. Hence, a fullwave simulator, specialized in Electromagnetic simulations at high frequency, CST Microwave Studio® 2014 [7] has been used for all simulation work in this paper. This simulation software work on a numerical method called Finite Integration Technique (FIT) [8], which discretizes the below mentioned Maxwell's equations [9] in integral form instead of differential ones,

$$
\begin{aligned}
& \oint \mathrm{E} \cdot d \mathrm{~L}=-\int_{s} \frac{\partial B}{\partial t} \cdot d \mathrm{~S} \\
& \oint \mathrm{H} \cdot d \mathrm{~L}=-\int_{s}\left(\mathrm{~J}+\frac{\partial \mathrm{D}}{\partial t}\right) \cdot d \mathrm{~S} \\
& \oint_{S} \mathrm{D} \cdot d \mathrm{~S}=\int_{v o l} \rho_{v} \cdot d v \\
& \oint_{S} \mathrm{~B} \cdot d \mathrm{~S}=0
\end{aligned}
$$

where $\mathbf{D}$ is Electric flux density, $\mathbf{E}$ is Electric Field Intensity, $\mathbf{B}$ is Magnetic Flux Density, $\mathbf{H}$ is Magnetic Field Intensity, $\mathbf{J}$ is conduction current density, $\rho_{v}$ is volume charge density and $\mathrm{dL}, \mathrm{dS}, \mathrm{d} v$ are differential length, surface area, volume respectively. This software breaks the calculation domain in small cuboidal cells, and solves the maxwell equations on all of its facets (boundaries).

A general Two-Port T network is shown in figure 1, with ABCD parameters as:

$$
\begin{aligned}
& A=1+\frac{Z_{1}}{Z_{3}} \\
& B=Z_{1+} Z_{2}+\frac{Z_{1} Z_{2}}{Z_{3}} \\
& C=\frac{1}{Z_{3}} \\
& D=1+\frac{Z_{2}}{Z_{3}}
\end{aligned}
$$


Table 1.Maximally flat low pass filter prototype element values

\begin{tabular}{|cccccc|}
\hline $\mathrm{N}$ & $g_{1}$ & $g_{2}$ & $g_{3}$ & $g_{4}$ & $g_{5}$ \\
\hline \hline 1 & 2.00001 & .0000 & & & \\
\hline 2 & 1.41421 & .41421 & .0000 & & \\
\hline 3 & 1.00002 & .00001 & .00001 & .0000 & \\
\hline 4 & 0.76541 & .84781 & .84780 & .76541 & .0000 \\
\hline
\end{tabular}

where $\mathrm{Z}$ is the impedance at or between the respective ports, and $Z_{0}$ is the line's characteristic impedance. This gives the impedance parameters as:

$$
\begin{aligned}
& Z_{11}=Z_{22}=\frac{A}{C}=-j Z_{0} \cot \beta l \\
& Z_{12}=Z_{21}=\frac{1}{C}=-j Z_{0} \csc \beta l
\end{aligned}
$$

where $\beta$ is the imaginary part of propagation constant, furthermore $l$ is length of the line. So, the series element comes out to be:

$$
Z_{11}-Z_{12}=-j Z_{0} \frac{\cos \beta l-1}{\sin \beta l}=j Z_{0} \tan \frac{\beta l}{2}
$$

whereas shunt element for $\mathrm{T}$ network is $Z_{12}$. When $l<\frac{\pi}{2}$, the series impedance is mainly positive reactance (Inductive) and shunt impedance is mainly negative reactance (Capacitive), giving out the equivalent circuit of figure 1(b) where,

$$
\begin{aligned}
& \frac{X}{2}=Z_{0} \tan \frac{\beta l}{2} \\
& B=\frac{1}{Z_{0}} \sin \beta l
\end{aligned}
$$

where $X$ is reactance, and $B$ is susceptance. So, the series Inductance may be replaced by high-impedance $\left(Z_{h}\right)$ line section, and shunt capacitance may be replaced by low-impedance $\left(Z_{l}\right)$ line section. Using scaling equations,

$$
\begin{aligned}
& L^{\prime}=\frac{R_{0} L}{w_{c}} \\
& C^{\prime}=\frac{C}{R_{0} w_{c}}
\end{aligned}
$$

Electrical lengths of Inductance and Capacitance can be calculated as: 


$$
\begin{array}{ll}
\beta l=\frac{C R_{0}}{Z_{h}} & \text { (Inductor) } \\
\beta l=\frac{C Z_{l}}{R_{0}} & \text { (Capacitor) }
\end{array}
$$

where $\mathrm{L}$ is inductance, and $\mathrm{C}$ is capacitance. Using table $\mathrm{I}$ [10], taking 2 nd order filter with $N=2, C_{1}=1.4142$, $L_{2}=1.4142, C_{3}=1.0000$, Step-Impedance [11] Microstrip Low-pass filter with pass-band in the $2.4 \mathrm{GHz}$ band along with stop-band in $5 \mathrm{GHz}$ band, possessing a low return loss around $2.5 \mathrm{GHz}$ is depicted in figure 2. A high high-low impedance ratio is used, for better approximations. Half of the filter, shown in Figure 2 is $180^{\circ}$ rotated, and the dimensions of lower and higher impedance lines are changed, and configured as an array structure as shown in figure 3 to obtain radiation in $2.4,5 \mathrm{GHz}$ bands. Figure 3 shows the planar antenna array obtained from modifications in stepped-impedance structure, it shows three possible bands of radiations, around $2.69 \mathrm{GHz}, 3.85 \mathrm{GHz}$, and $5.5 \mathrm{GHz}$. The first band needs to be shifted towards lower frequency, and the middle one is unwanted as it will reduce the radiation aperture for the two bands of WLAN network. These two problems, as well as lower return loss at required frequency bands will be obtained by incorporating this design with CSRRs, which are discussed in following sections.

CSRRs exhibit negative permittivity [5]. Here it works mainly as a resonator instead of a radiator, providing more control over the coupling. It acts like a tank L-C Resonator circuit [12] as shown in figure 4. The Capacitive coupling is mainly obtained from ring slots, while the outer ring's split provides magnetic coupling.
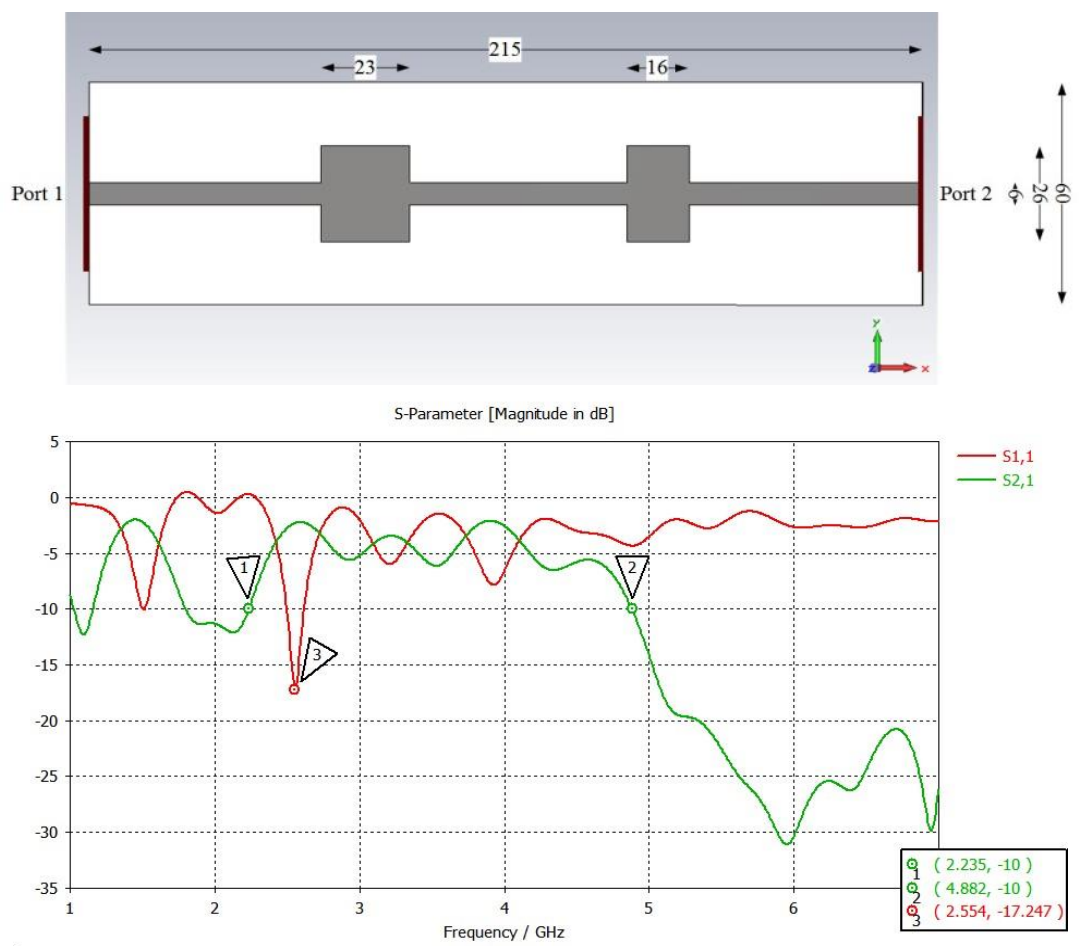

Fig.2.(a) Stepped-Impedance Low Pass Filter design. (b) S-Parameters 


\section{Antenna Design}

Figure 5 shows the planar antenna array loaded with Complementary Split Ring Resonators. This design is symmetrical across y-axis, giving symmetrical radiation in H-plane. The structure is suspended above the ground plane at the height of $1 \mathrm{~mm}$. All the measurements and angles have been depicted in the figure itself. Stepped-Impedance structure is used and modified as an array, for better directivity; which radiated in $2.5 \mathrm{GHz}$, $5 \mathrm{GHz}$ bands, in addition to an unwanted band. This shortcoming was removed by using CSRRs, which act as a resonator providing more maneuverability over mutual coupling, it also results in better reflection coefficients in both the required bands, and left shifting the lower band to radiate in the exact $2.4 \mathrm{GHz}$ band.

\section{Results and Discussions}

Figure 6 (a) depicts the Scattering parameter $S_{11}$, this reflections coefficient shows that very less power is reflected back from $2.423 \mathrm{GHz}$ to $2.5283 \mathrm{GHz}$, with the minimum going to $-19.11 \mathrm{~dB}$ at $2.472 \mathrm{GHz}$, giving a potential bandwidth of $105.3 \mathrm{MHz}$ for the first band of WLAN i.e. $2.4 \mathrm{GHz}$ band. For the second band of WLAN, this antenna again shows very low return loss from $5.2701 \mathrm{GHz}$ to $5.8879 \mathrm{GHz}$, likewise the minimum going to $-24.378 \mathrm{~dB}$ at $5.568 \mathrm{GHz}$, giving a potential bandwidth of $617.8 \mathrm{MHz}$ for $5 \mathrm{GHz}$ band. Using equation 18 [13], most of the power feed to antenna, in these two bands will be utilized by the antenna, and a very less power will be reflected back, giving out very low Standing Wave Ratio of 1.02485 and 1.007325 at $2.472 \mathrm{GHz}$ and $5.568 \mathrm{GHz}$ respectively.

$$
S W R=\frac{1+|\Gamma|}{1-|\Gamma|}
$$

where SWR is Standing Wave Ratio for the wave with the reflection coefficient $\Gamma$. Figure 6 (b) and (c) shows the absolute farfield directivity, at a fixed azimuthal angle of $90^{\circ}$ in both the bands at $2.472 \mathrm{GHz}$ as well as $5.568 \mathrm{GHz}$. The antenna has a good directivity because of array type structure, the first band shows a directivity of $11.6 \mathrm{dBi}$ at polar angle of $22^{\circ}$ with a $3 \mathrm{~dB}$ angular width of $25.2^{\circ}$. The second band shows a directivity of $11.3 \mathrm{dBi}$ at polar angle of $25^{\circ}$ with a $3 \mathrm{~dB}$ angular width of $27.1^{\circ}$. Since, the design is symmetrical

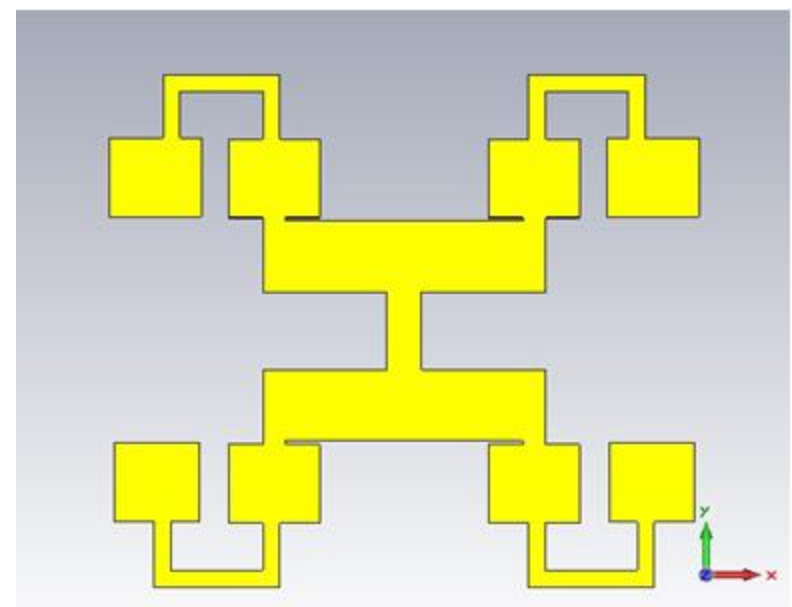




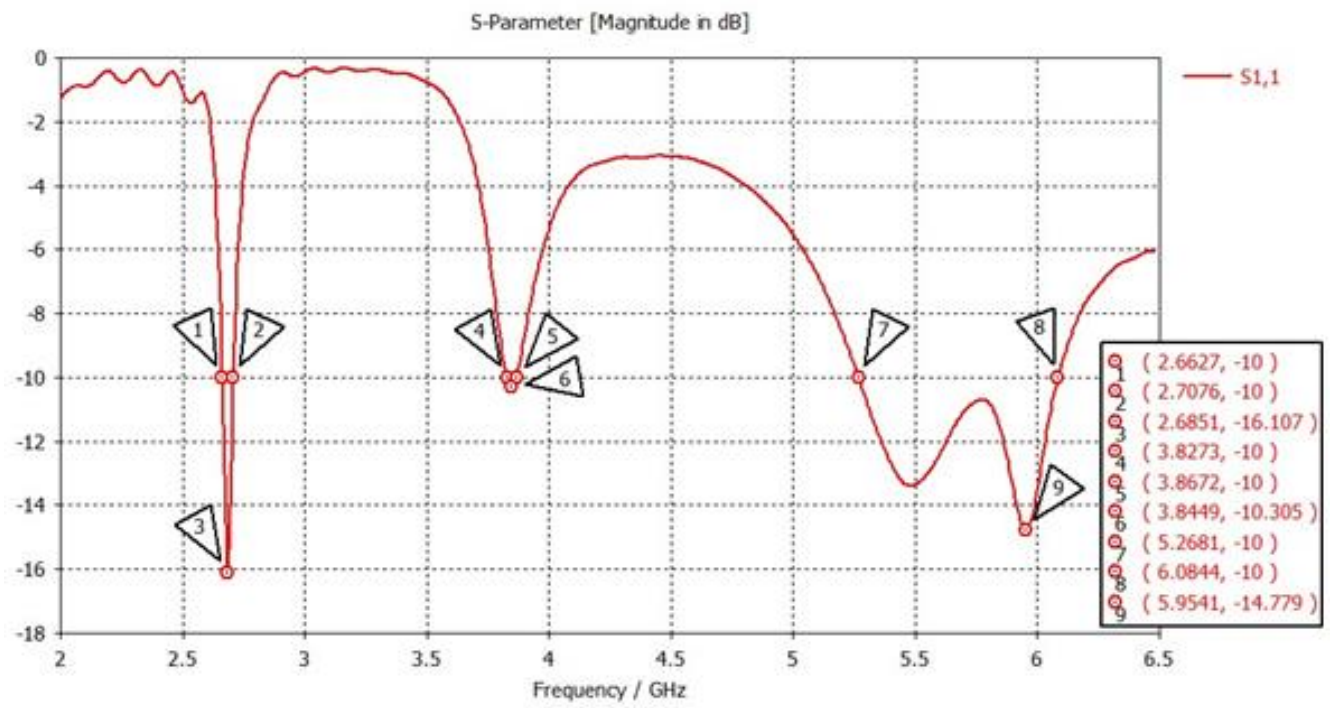

Fig.3.(a) Planar array design without CSRRs. (b) Reflection Coefficient.
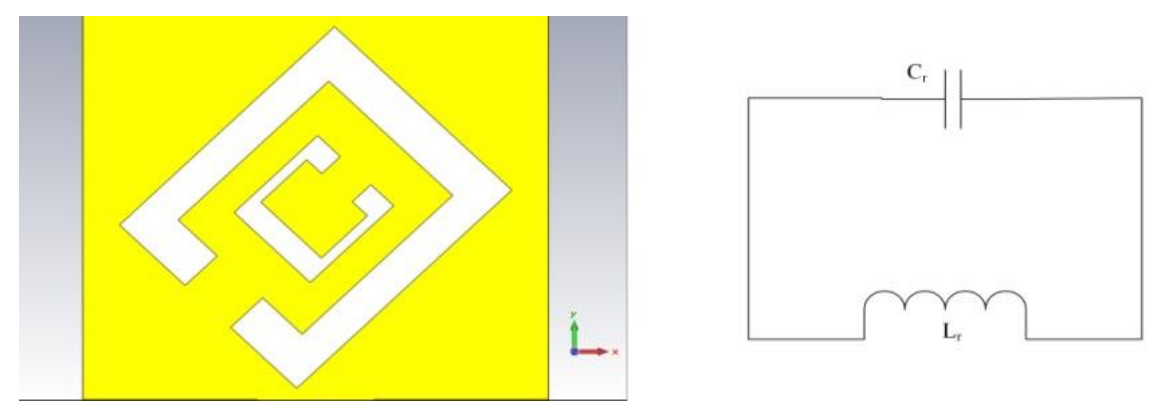

Fig.4.CSRR and its equivalent circuit

across y-axis, this gives a symmetrical radiation pattern in H-plane, in both the bands. Usage of Complementary Split Ring Resonators in array structure, has improved mainly four result aspects of the design, one is further reduction of return loss in first WLAN band $2.4 \mathrm{GHz}$, likewise second is also further reduction of return loss in second WLAN band $5 \mathrm{GHz}$, third is removal of unwanted radiation at $3.845 \mathrm{GHz}$ giving forth a better radiation efficiency in both the radiating bands of WLAN, fourth is left shifting the $2.4 \mathrm{GHz}$ band to radiate suitably in proper band. As the antenna is also covering the band for Bluetooth $2.4 \mathrm{GHz}$ to $2.5 \mathrm{GHz}$, this configuration can be used for Bluetooth applications as well. 


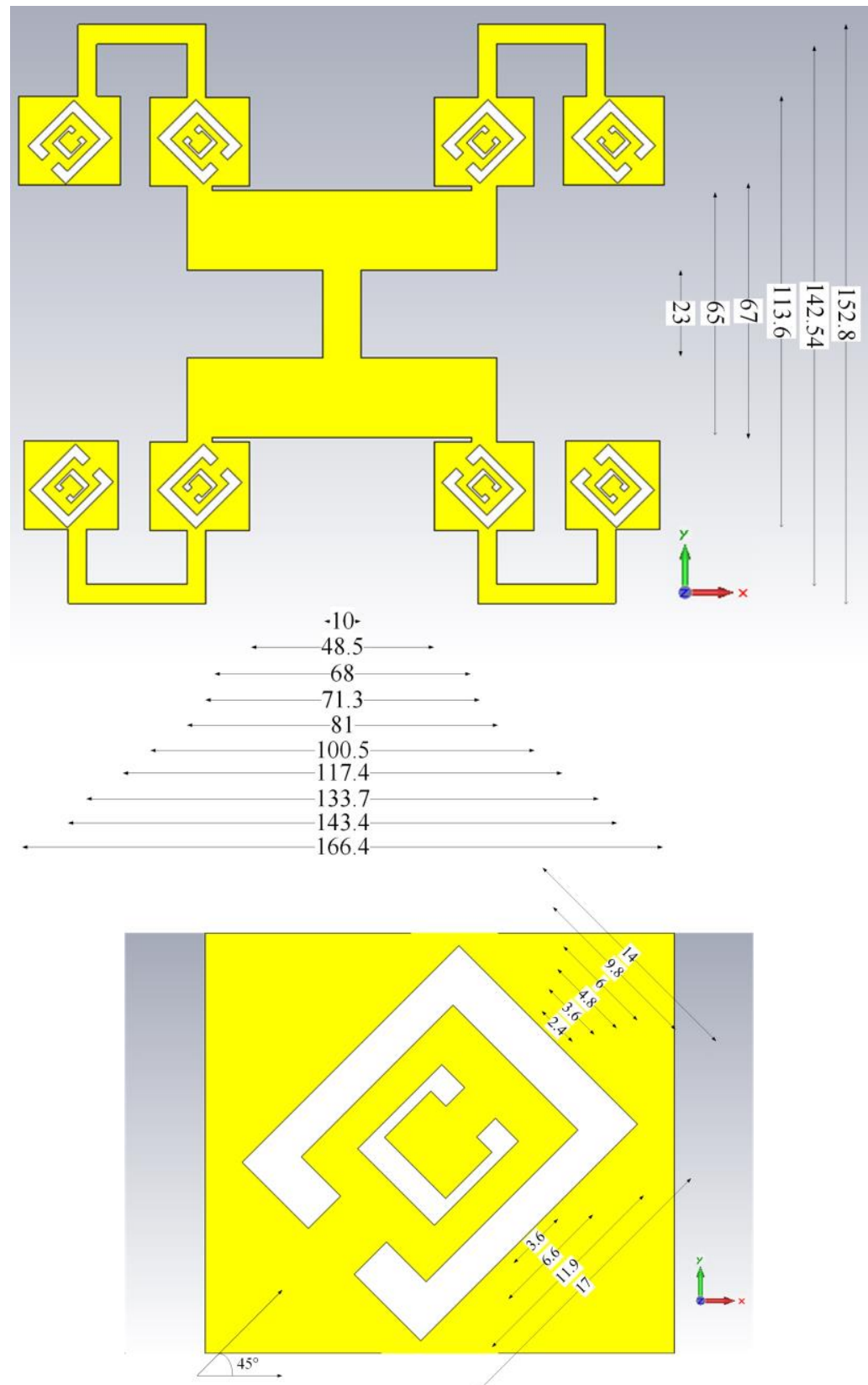

Fig.5.(a) Schematic diagram of Rotated-Stepped-Impedance array loaded with CSRRs (b) Schematic diagram of loaded CSRR.

\section{Conclusions}

The antenna is the planar dual band microstrip patch antenna for WLAN (WiFi) applications. It is based on a array structure, having stepped impedance filter, loaded with Complementary Split Ring Resonators, thus 
giving a good directivity and low return losses. It can be used commercially as its giving good result and the structure is robust, have a good mechanical tolerance, compact, and cost effective.

\section{Acknowledgement}

The authors sincerely thank Dr. Rajesh Khanna, Professor at Thapar University Patiala, India, for the support he has provided by establishing Antenna Research Lab, and giving his valuable guidance during the course of research. The authors also thank $\mathrm{L}^{\mathrm{A}} \mathrm{T}_{\mathrm{E}} \mathrm{X}$ community for its typesetting support.

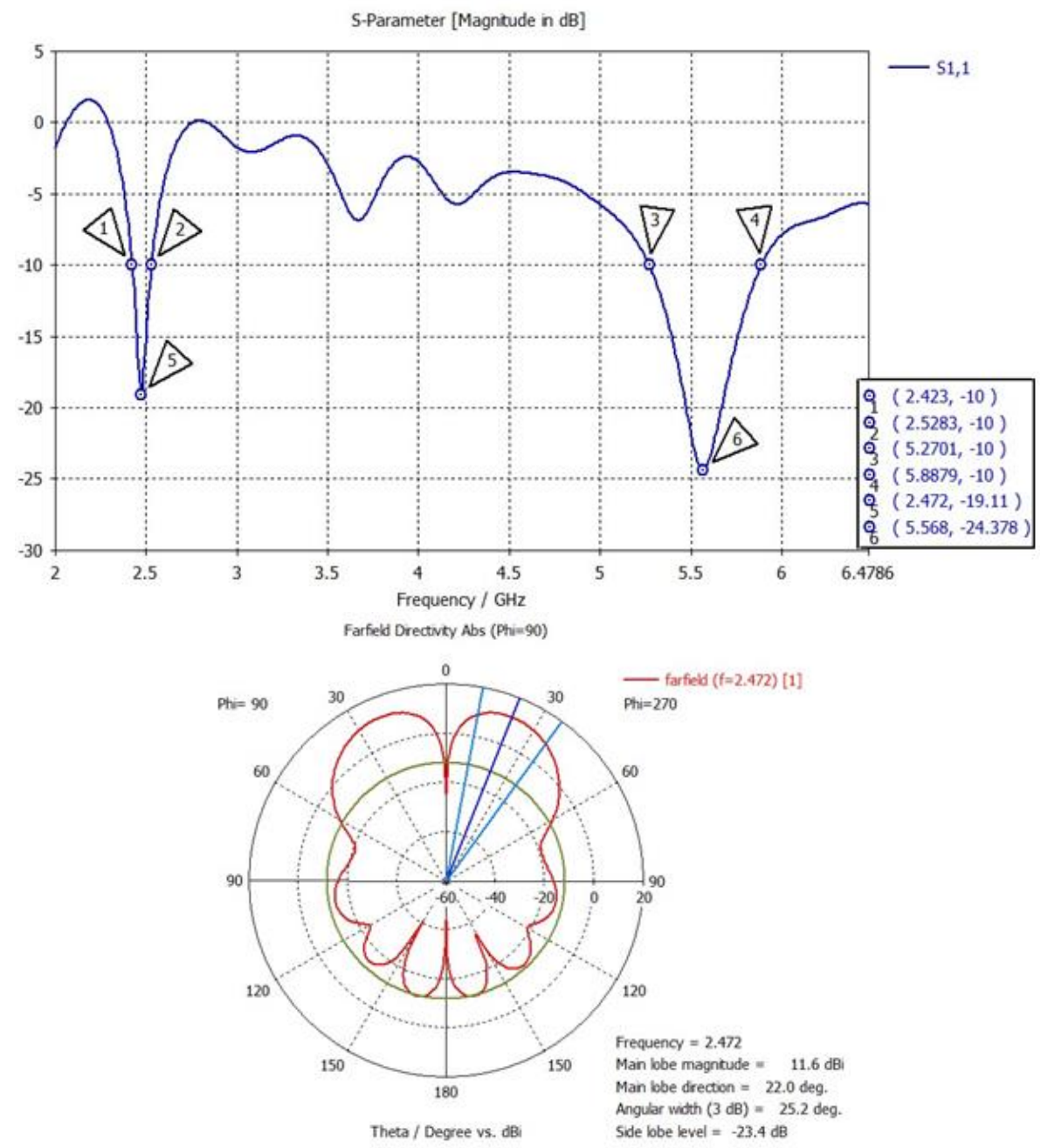




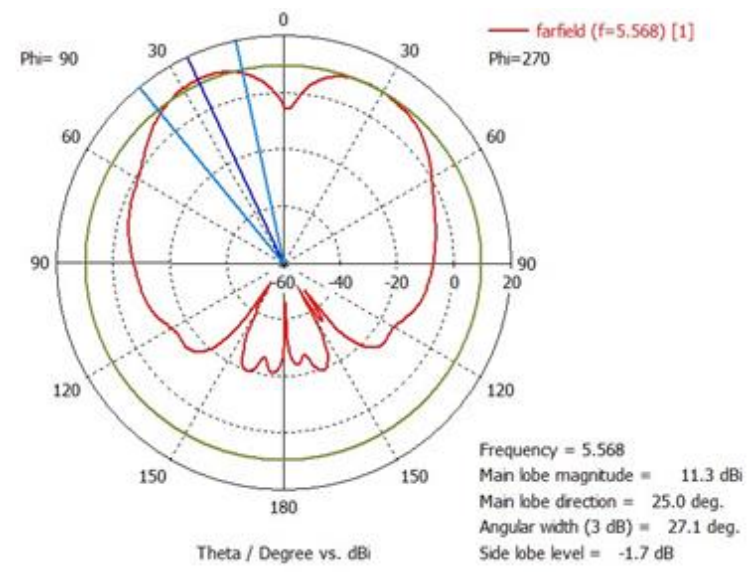

Fig.6.(a) Reflection Coefficient. (b) Farfield at first frequency band-2.4 GHz. (c) Farfield at second frequency band-5 GHz

\section{References}

[1] (2015) The IEEE Standards Association website. [Online]. Available: http://standards.ieee.org/about/get/802/802.11.html

[2] J. Kaur and R. Khanna, "Development of dual-band microstrip patch antenna for wlan/mimo/wimax/amsat/wave applications," Microwave and Optical Technology Letters, vol. 56, no. 4, pp. 988-993, 2014.

[3] J. Kaur, R. Khanna, and M. V. Kartikeyan, "Optimization and development of o-shaped triple-band microstrip patch antenna for wireless communication applications," IETE Journal of Research, vol. 60, no. 2, pp. 95-105, 2014.

[4] A. Saleh and S. Abdullah, "A novel design of patch antenna loaded with complementary split-ring resonator and 1-shape slot for (wimax/wlan) applications," International Journal of Wireless and Microwave Technologies, vol. 4, no. 3, pp. 16-25, October 2014.

[5] F. Falcone, T. Lopetegi, J. Baena, R. Marques, F. Martin, and M. Sorolla, "Effective negative- epsiv; stopband microstrip lines based on complementary split ring resonators," Microwave and Wireless Components Letters, IEEE, vol. 14, no. 6, pp. 280-282, June 2004.

[6] K. F. Lee and K. M. Luk, "Microstrip antenna array," in Microstrip Patch Antennas.Imperial College Press, 2011, ch. 17, pp. 487-515.

[7] (2015) The CST Microwave Studio website. [Online]. Available: https://www.cst.com/Products/CSTMWS

[8] T. Weiland, "Finite integration method and discrete electromagnetism," in Computational Electromagnetics, W. H. R. H. P. M. C. Carstensen, S. Funken, Ed. Springer Berlin Heidelberg, 2003, ch. 3, pp. 183-198.

[9] W. H. Hayt and J. A. Buck, "Time-varying fields and maxwell's equations," in Engineering Electromagnetics. Tata McGraw Hill Education Private Limited, 2012, ch. 10, pp. 306-321.

[10] L. Y. George L. Matthaei and E. M. T. Jones, "Low-pass prototype filters obtained by network synthesis methods," in Microwave Filters, ImpedanceMatching Networks, and Coupling Structures. Artech House, Inc., 1980, ch. 4, pp. 83-162.

[11] D. M. Pozar, "Microwave filters," in Microwave Engineering. John Wiley \& Sons, Inc., 2012, ch. 8, pp. 380-443. 
[12] J. Baena, J. Bonache, F. Martin, R. Sillero, F. Falcone, T. Lopetegi, M. Laso, J. Garcia-Garcia, I. Gil, M. Portillo, and M. Sorolla, "Equivalent-circuit models for split-ring resonators and complementary split-ring resonators coupled to planar transmission lines," Microwave Theory and Techniques, IEEE Transactions on, vol. 53, no. 4, pp. 1451-1461, April 2005.

[13] (2015) The Wikipedia website. [Online]. Available: https://en.wikipedia.org/wiki/SWR

\section{Authors' Profiles}

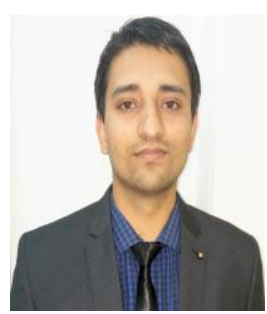

Deepak (IEEE member) has completed his M.E. in "Wireless Communications" from "Electronics and Communication Engineering Department", Thapar University, Patiala, Punjab, India in 2015. His B.E. is in "Electronics and Communication Engineering" from Panjab University, Chandigarh, in 2011. He can be contacted at deepak08wc@gmail.com.

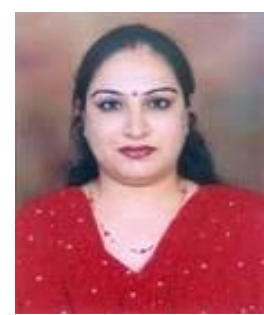

Dr. Jaswinder Kaur is a lecturer at "Electronics and Communication Engineering Department", Thapar University, Patiala, Punjab, India. She received her Ph.D. degree from "Department of Electronics and Communication Engineering", Thapar University, Patiala, Punjab, India in 2014. Her M.Tech. and B.Tech. degrees are from Punjab Technical University, Jalandhar, in 2009 and 2005 respectively. She has been in the profession of teaching since July 2005. Her research interests include the Analysis and Design of Antennas and Wireless Communication Systems. She has 24 publications in national and international peer reviewed journals/conferences, to her credit. She can be contacted at jaswinder.kaur@thapar.edu

How to cite this paper: Deepak, Jaswinder Kaur,"Dual Band High Directivity Microstrip Patch Antenna Rotated-Stepped-Impedance Array Loaded with CSRRs for WLAN Applications", International Journal of Wireless and Microwave Technologies(IJWMT), Vol.6, No.4, pp.1-11, 2016.DOI: 10.5815/ijwmt.2016.04.01 